\section{Obturación en endodoncia - Nuevos sistemas de obturación: revisión de literatura}

Giudice-García A, Torres-Navarro J. Obturación en endodoncia - Nuevos sistemas de obturación: revisión de literatura. Rev Estomatol Herediana. 2011; 21(3):166-174.

RESUMEN

El objetivo de esta revisión fue de recopilar la información correspondiente a las bases y criterios para la obturación del sistema de conductos radiculares así como de los nuevos sistemas de obturación disponibles a nivel mundial, evaluando sus características, ventajas y desventajas.

Palabras clave: OBTURACIÓN DEL CONDUCTO RADICULAR / MATERIALES DE OBTURACIÓN DEL CONDUCTO RADICULAR / GUTAPERCHA.

Endodontics filling - New filling systems: review of the literature ABSTRACT

The aim of this review was to gather information on the basis and criteria for filling the root canal system and the new sealing systems available worldwide, evaluating their characteristics, advantages and disadvantages.

Key words: ROOT CANAL OBTURATION / ROOT CANAL FILLING MATERIALS / GUTTAPERCHA.

\section{Andrea Giudice García ${ }^{1}$ John Torres Navarro²}

\author{
'Residente del Programa de Especilaización en \\ Endodoncia. \\ 2Docente del Departamento Académico de Cínica \\ Estomatológica. Facultad de Estomatología. \\ Universidad Peruana Cayetano Heredia.
}

\section{Correspondencia}

John Torres Navarro

Calle Francisco de Orellana 171 - Lima 12, Perú. Teléfono: 3490779

e-mail: john.torres@upch.pe

Recibido : 14 de abril de 2011

Aceptado : 5 de setiembre de 2011

\section{Introducción}

De acuerdo a la asociación americana de endodoncia (AAE), una obturación adecuada se define y se caracteriza por el llenado tridimensional de todo el conducto radicular, lo más cercano posible de la unión cemento-dentinaria. La obturación es la última etapa operatoria del tratamiento de conductos radiculares, y tiene valor fundamental en el éxito a mediano y largo plazo, por lo que su objetivo final es la obturación completa del sistema de conductos radiculares para lograr la preservación del diente como una unidad funcional sana (1).

Las características ideales de la obturación del sistema de conductos radiculares son las siguientes (2):

- Debe ser realizada de forma tridimensional para lograr prevenir la percolación y microfiltración hacia los tejidos periapicales del contenido del sistema de conducto radicular y también en sentido contrario.

- Utilizar la mínima cantidad de cemento sellador, el cual debe ser biológicamente compatible al igual que el material de relleno sólido, y químicamente entre sí para establecer una unión de los mismos y así un selle adecuado.

- Radiográficamente el relleno debe extenderse lo más cerca posible de la unión cemento dentinal y observarse denso. El conducto obturado debe reflejar una conformación que se aproxime a la morfología radicular. Así mismo, debe mostrar una preparación continua en forma de embudo y estrecha en el ápice, sin excesiva eliminación de estructura dentinaria en cualquier nivel del sistema del conducto, porque el material obturador no fortalece la raíz ni compensa la pérdida de dentina.

Es por esto la importancia de los diversos sistemas de obturación que aparecen y cumplan estas características para el éxito de nuestro tratamiento de conductos radiculares.

\section{Materiales de obturación del conducto radicular}

Se ha realizado una clasificación de los materiales de obturación en materiales en estado sólido (conos de gutapercha y plata) y materiales en estado plástico (cementos y pastas). A pesar de que esta clasifica- ción es muy objetiva es necesario en los procedimientos endodónticos lograr un binomio ideal entre el material sólido y el plástico como asociación imprescindible en la obturación del sistema de conductos radiculares (3). Así, el método de obturación más aceptado actualmente emplea un núcleo sólido o semisólido, como lo es la gutapercha, y un cemento sellador del conducto radicular (4).

\section{Gutapercha.}

El material más ampliamente utilizado y aceptado para la obturación de los conductos preparados es la gutapercha Históricamente, la gutapercha ha demostrado ser el material de elección para el mejor llenado del conducto, desde la corona hasta la porción apical (1).

La gutapercha (transpoliisopreno) puede presentarse en tres formas distintas: dos formas esteáricas cristalinas ( $\alpha$ y $\beta$ ) y una forma amorfa o fundida. Las tres forman parte de la obturación de conductos radiculares. Las puntas convencionales de gutapercha están fabricadas de fase $\beta$, que se transforma en fase $\alpha$ cuando se calienta 
a $42-49^{\circ} \mathrm{C}$. En el calentamiento continuado se pierde la forma cristalina para proporcionar una mezcla amorfa a $59^{\circ} \mathrm{C}$ (las temperaturas exactas dependen de la marca utilizada). Estas transformaciones de fase están asociadas con cambios volumétricos, con una relevancia obvia en la obturación de los conductos radiculares. La gutapercha calentada a una temperatura muy alta se contrae más al enfriarse (5).

Sus desventajas, como la falta de rigidez y la adhesividad o la facilidad para salir del lugar al ser presionado no disminuyen las ventajas. Han buscado diversas alternativas para reemplazar a la gutapercha pero ningún otro material ha mostrado estar en condiciones de sustituirla y permanece como modelo de calidad para la obturación (1).

\section{Resilon ${ }^{\circledR}$.}

Recientemente se propusieron sistemas de obturación a base de resina, presentando como alternativa a la gutapercha el Resilon ${ }^{\circledR}$ (Pentron clinical technologies, Wellingford, CT, EEUU), un poliuretano industrial adaptado para uso endodóntico. El Resilon ${ }^{\circledR}$ es similar a la gutapercha en sus propiedades físicas y permite obturar el sistema de conductos con las mismas técnicas (condensación lateral activa, condensación vertical con calor e inyección termoplástica) (6).

Los sistemas a base de Resilon ${ }^{\circledR}$ incorporan a la resina de llenado (equivalente a la gutapercha) un cemento sellador, denominado Epiphany ${ }^{\circledR}$ (Pentron clinical technologies, Wellingford, CT, EEUU), capaz de unirse a la dentina y a la resina del núcleo (Resilon). La SybronEndo (Glendora, CA, EEUU) distribuye este sistema con la denominación de RealSeal ${ }^{\circledR}$. La principal característica de estos materiales, según sus creadores, es que el sellador de resina se uniría a las paredes del conducto y al material de llenado, formando así una obturación en bloque (monobloque), que en principio reduciría la filtración marginal y proporcionaría mayor resistencia del diente, como lo muestran algunos artículos (7-9). Además, las características de manipulación son muy similares a las de la gutapercha (salvo la temperatura de plastificación, que debe ser más baja que la utilizada en las técnicas termoplásticas), pues su uso es relativamente simple. Biológicamente presenta excelentes resultados cuando fue evaluado microscópicamente en la región periapical de dientes de perro (7).

\section{Técnicas de obturación}

Las técnicas que actualmente tenemos a disposición para realizar la obturación del sistema de conductos radiculares varían según la dirección de compactación de la gutapercha (lateral o vertical) y la temperatura que debe aplicarse, fría o caliente (plastificada). Las técnicas de obturación son diversas de las que destacan las siguientes (1):

- Condensación lateral activa.

- Condensación vertical (gutapercha caliente).

- Gutapercha en frio (Gutta Flow).

- Gutapercha termoplastificada inyectable.

- Compactación termomecánica o termocompactación de la gutapercha.

- Conductores de núcleo o centro solido, envueltos con gutapercha alfa.

Condensación lateral activa en frío.

La técnica de la condensación lateral de gutapercha es la técnica más conocida y utilizada para obturar los conductos radiculares. Des- pués de la preparación del conducto, se selecciona el cono principal; se confirma su posición en la longitud de trabajo mediante la radiografía (10). Una vez ajustado el cono de gutapercha principal después de su remoción debemos eliminar el barro dentinario (Smear Layer) utilizando solución de EDTA o acido cítrico. Después de seleccionar el cono principal y el espaciador con el conducto radicular sin Smear Layer seco, colocamos el cemento endodóntico (1).

Se seca el conducto radicular y se prepara el cemento obturador. El siguiente paso es colocar los conos accesorios que deben ser posicionados lo más próximos al ápice radicular. El espacio creado con la retirada del espaciador debe rellenarse inmediatamente con un cono accesorio de diámetro análogo al del espaciador. Este procedimiento se repite hasta que el espaciador no encuentre espacio para penetrar más allá del tercio cervical (10).

\section{Condensación vertical.}

Se propuso a partir de la premisa que la compactación de la gutapercha calentada permitiría obtener mejor adaptación del material a las irregularidades de los conductos radiculares y se podrían obturar de forma más previsible conductos laterales, ramificaciones e istmos (1).

Se utiliza un cono de gutapercha con conicidad ligeramente inferior a la de la preparación del conducto, porque de esta manera, el ajuste del cono de gutapercha se producirá seguramente en el tope apical y no en otras partes del conducto (11).

La técnica se basa en el calentamiento del cono de gutapercha y su posterior compactación en sucesivas aplicaciones. Por lo tanto, hay que seleccionar varios 
condensadores, de diferentes diámetros para que actúen en las diferentes partes del conducto.

Una vez seleccionado el cono de gutapercha y los condensadores de inicia la obturación. El cono de gutapercha principal recubierto por el cemento sellador, se coloca en el conducto radicular. Se elimina la parte del cono de gutapercha que sobresale del conducto con un condensador calentado en la llama o con el dispositivo Touch and Heat ${ }^{\circledR}$ (SybronEndo, Orange, CA, EEUU) (1).

Posteriormente, se calienta la gutapercha mas coronal parte de la cual se elimina también al calentarla, y se ejerce presión sobre la gutapercha en dirección apical con el condensador sin calentar. Se repite sucesivamente esta etapa utilizando condensadores cada vez más finos, pues se trabaja cada vez más cerca de la parte apical de la preparación. Cuando faltan cuatro milímetros de la longitud real de trabajo, se considera terminada la primera parte de la obturación. Para obturar el resto del conducto pueden utilizarse técnicas como la inyección de gutapercha termoplástica, la técnica hibrida de Tagger, la condensación lateral activa o el sistema Thermafill@ (12).

Condensación vertical de onda continúa.

En los años noventa, Buchanan introdujo el System B ${ }^{\circledR}$ (Sybron Endo, Orange, CA, EEUU) para realizar la condensación vertical de manera más simple. La técnica presenta una serie de diferencias con relación a la técnica clásica de condensación vertical. Cuando se utiliza el System $B \circledR$, el mismo instrumento es transportador de calor y condensador de gutapercha. A diferencia de la técnica tradicional, en la técnica de onda continua, se realiza toda la condensación vertical en una única etapa (1).

El System $B{ }^{\circledR}$ consta de cinco condensadores de diferentes conicidades, de $4 \%, 6 \%, 8 \%, 10 \%$, $12 \%$ y uno con diámetro apical de $0,5 \mathrm{~mm}$. El primer paso de la técnica corresponde a la selección del condensador que será utilizado. Hay que seleccionar el condensador de mayor conicidad para que llegue hasta $5-7 \mathrm{~mm}$ antes de la longitud real de trabajo. De acuerdo con diversos autores se obtienen mejores resultados cuando el condensador llega hasta 3-5 mm antes de la longitud real de trabajo (13-16).

Después de secar el conducto radicular se introduce el cono de gutapercha, recubierto con cemento, hasta la longitud real de trabajo. Se programa el System B ${ }^{\circledR}$ para la temperatura de $200^{\circ} \mathrm{C}$ y se calienta el condensador para cortar la gutapercha que sobresale del conducto. Posteriormente, con un único movimiento se calienta y condensa la gutapercha en dirección apical con el condensador calentado a $200^{\circ} \mathrm{C}$. Cuando el condensador llega a $3 \mathrm{~mm}$ del punto hasta donde debe penetrar, se deja de aplicar calor y se ejerce presión apical hasta que el condensador llegue a aproximadamente un milímetro del punto de penetración máximo predeterminado y se mantiene la presión en dirección apical durante unos diez segundos.

El condensador debe quedar a $1 \mathrm{~mm}$ de distancia de donde esté sujeto, de lo contrario no condensaría la gutapercha y podría provocar una fractura vertical al ejercer fuerza sobre la paredes del conducto radicular. Para retirar el condensador después de condensar la gutapercha, hay que calentarlo durante un segundo y se retira en dirección coronal. En conductos ovalados puede colocarse un cono auxiliar, además del cono principal, para aumentar la cantidad de gutapercha en el interior del conducto y permitir la generación de fuerzas hidráulicas. (1)

Técnicas con gutapercha en frío.

Según el fabricante GuttaFlow ${ }^{\circledR}$ es un sistema completamente nuevo de llenado de conductos radiculares, que combina dos productos en uno: la gutapercha en forma de polvo con un tamaño de partícula inferior a 30 micras y sellador. Este nuevo sistema de relleno con gutapercha fría de flujo libre-percha utiliza un sistema de aplicación que permite un procedimiento absolutamente simple, seguro e higiénico.

GuttaFlow ${ }^{\circledR}$ es la primera Gutapercha no caliente de flujo libre que no se contrae. Permite una gran facilidad de manejo como punto principal (la condensación no es necesaria) tiene excelentes propiedades de flujo que permiten una optima distribución en el canal radicular. Es extremadamente biocompatible y permite la preparación de un buen poste el cual se puede retirar fácilmente durante el retratamiento. Además asegura un cierre muy ajustado del conducto radicular y es radiopaco para una correcta evaluación radiográfica (17).

Según las indicaciones del fabricante: GuttaFlow ${ }^{\circledR}$, tiempo de trabajo 10-15 minutos, tiempo de fraguado 25-30 minutos. GuttaFlow FAST ${ }^{\circledR}$, tiempo de trabajo 4-5 minutos, tiempo de fraguado de 2530min.

GuttaFlow ${ }^{\circledR}$ reduce el tiempo de trabajo para la sesión de tratamiento de conducto. Esta ventaja en el tiempo se muestra durante la obturación de uno o dos canales radiculares. El exceso de material 
al abrir la cavidad se puede quitar más rápidamente además del curado rápido. Una pasta temporal se puede colocar junto a GuttaFlow FAST ${ }^{\circledR}$ en la sesión de tratamiento.

En su estudio sobre la capacidad de sellado del Guttaflow ${ }^{\circledR}$ en conductos ovalados utilizando un modelo in vitro de filtración polimicrobiana, obtuvieron que este producto tenía mayor filtración frente a otros como: el cemento AH plus ${ }^{\circledR}$, el cemento sellador $\mathrm{EWt}{ }^{\circledR}$ y el cemento Roekoseal ${ }^{\circledR}$, (todos los sistemas estudiados utilizaron la técnica de cono único). Estos autores expusieron su muestra a saliva humana durante nueve semanas para ver la filtración bacteriana existente. Después de este periodo llegaron a la conclusión que no encontraron diferencias significativas entre los grupos de AH plus ${ }^{\circledR}$ y cemento EWt ${ }^{\circledR}$, mientras que los grupos formados por Guttaflow ${ }^{\circledR}$ y Roekoseal ${ }^{\circledR}$ presentaban gran filtración, presentando el primero de estos un filtración total (18).

Técnicas de inyección de gutapercha termoplástica.

La diferencia entre las técnicas de inyección de gutapercha termoplástica y la anterior es que el calentamiento de la gutapercha se realiza fuera del conducto radicular. Las técnicas de inyección de gutapercha termoplástica se indican cuando (1):

- El conducto es muy amplio, como en los diente con ápices inmaduros en los que se obtura previamente la parte apical con MTA

- En conductos radiculares en forma de $\mathrm{C}$

- En dientes con reabsorción interna

El sistema también es de gran utilidad para obturar los tercios medio y coronal de conductos en los que se obtura el tercio apical con condensación vertical y también para obturar la totalidad de conducto radicular $(16,17)$. Un problema de las técnicas de inyección de la gutapercha termoplástica es la falta de control apical. Por eso en muchos casos se utiliza para complementar otras técnicas utilizadas para obturar la porción apical del conducto (3).

\section{Obtura $11{ }^{\circledR}$.}

El sistema Obtura II ${ }^{\circledR}$ (Obtura Spartan, Fenton MO, EEUU) utiliza una pistola cargada con un cartucho de gutapercha que se calienta a una temperatura de hasta $170^{\circ} \mathrm{C}$. Se utilizan agujas aplicadoras de plata para introducir la gutrapercha las cuales están conectadas a la pistola. Esta aguja debe llegar entre 3-5 mm de la preparación apical utilizando una técnica segmentada, llevando sucesivas cantidades de gutapercha al interior del conducto radicular para posteriormente proceder a su condensación, o una técnica en la que se introduce la gutapercha en toda la extensión del conducto de una sola vez.

Se coloca cemento sellador en el interior del conducto, con cualquiera de las técnicas anteriormente citadas. En seguida se compacta la gutapercha en dirección apical con un único condensador seleccionado previamente. Es importante realizar una compactación correcta, pues la gutapercha termoplástica experimenta contracción al enfriarse. Una vez terminada la compactación, se aplica nuevamente 3-4mm de gutapercha y se continua con la compactación mediante un condensador de mayor diámetro. Hay que repetir estos pasos hasta que el conducto quede completamente obturado (1).
En la técnica de obturación del conducto en una sola etapa, inyectamos la gutapercha a 3-5 mm de la preparación apical (tope) y la aguja va retrocediendo a medida que se llena el conducto. Una vez obturado por completo, se presiona en dirección apical con un condensador, hasta que la gutapercha se enfría, compensando así parcialmente la contracción de la gutapercha, que puede ser de hasta el $2 \%$ del volumen (19).

Compactación termomecánica o termocompactación de la gutapercha.

El concepto de termoplastificación de la gutapercha se introdujo en 1980. Inicialmente, el compactador de McSpadden era un instrumento similar a una lima tipo Hedstroem invertida. Se montaba el instrumento en un contraángulo y después se introducía en el conducto radicular, girando entre $8000 \mathrm{y}$ 10000 rpm (1).

Con estas velocidades, el calor generado por la fricción plastificaba la gutapercha compactando el material en sentido apical, mientras que el condensador era impulsado en sentido coronal. Sin embargo la fragilidad y la fractura de los instrumentos, como también la posibilidad de sobre obturación, además de la dificultad para dominar la técnica, impidieron que tuviese mucha difusión entre los endodoncistas. No obstante, con el surgimiento de diferentes configuraciones para los instrumentos y nuevas formas de aplicación su uso fue generalizándose (1).

En Europa, la firma Maillefer modificó el instrumento tipo lima Hedstroem invertida y lo denominó Gutta Condensor ${ }^{\circledR}$ y la Zipperer lo denominó Engine Plugger ${ }^{\circledR}$, este último se parece más a una lima tipo $\mathrm{K}$ invertida. Recientemente se in- 
trodujeron variaciones y estos instrumentos comenzaron a confeccionarse con aleación de niquel titanio, para que tuviesen más flexibilidad, como por ejemplo los condensadores Pac Mac ${ }^{\circledR}$ (Analytic-Endodontics, Glendora, CA, EEUU).

Numerosos estudios evaluaron la eficacia de esa técnica de obturación del conducto radicular. Los hallazgos fueron muy diversos pero parecían ser positivos. Las técnicas eran rápidas, con economía de conos de gutapercha, el sellado del sistema de conductos parece adecuado y radiográficamente había buena adaptación a las irregularidades anatómicas. Los problemas incluían el sobrepaso del material de obturación, excavaciones en las paredes del canal, fractura del termocondensador, posibilidad de fracturas verticales y destrucción de dentina $(20,21)$.

Algunos investigadores identificaron el potencial de esta técnica para producir calor excesivo y nocivo, aumentando la temperatura externa radicular (22) lo que podría provocar lesión en los tejidos periodontales de soporte por el súper calentamiento, causando reabsorción y anquilosis. Sin embargo, si el calor que se transmite a los tejidos de soporte es suficiente intenso, el daño producido a estos tejidos podría ocurrir también con otras técnicas en las que se utilice gutapercha calentada $(23,24)$.

Velocidades más lentas y la colocación de la gutapercha a una temperatura baja se necesitan para minimizar las altas temperaturas y el estrés en el sistema de conductos radiculares durante la compactación rotatoria. El uso de velocidades mayores que las recomendadas puede producir un sellado más pobre. La preparación esmerada del con- ducto y la profundidad de la penetración del condensador rotatorio ayudan a evitar posibles accidentes operatorios con el uso de esta técnica; de cualquier forma, se recomienda el aprendizaje in vitro (1).

Tagger et al. Recomendaron una técnica híbrida, en la que el tercio apical del conducto se obtura con condensación lateral activa en frio y el remanente con el termo condensador. La técnica resuelve el control en el límite apical de obturación inherente de la termocondensación. La técnica resuelve el control en el límite apical de obturación inherente de la termocondensación, acelera la obturación y reduce la cantidad de gutapercha. Puede obtenerse una obturación homogénea y eficaz, a pesar de que los resultados son variables. Es una técnica valida que complementa muy bien la condensación lateral (25).

Conductores de núcleo o centro sólido, envueltos con gutapercha alfa Sistema Thermafil.

En 1978 se presentó un método simple de distribución o aplicación de la gutapercha termoplastificada en un conducto debidamente preparado y confeccionado. El desarrollo inicial de este sistema consistía en el uso de portadores (carriers) metálicos para la aplicación de la gutapercha blanda. Este sistema posibilitaba la distribución del material con control apical razonable y con uniformidad de la densidad, lo que permite fácil adaptación a las paredes del conducto y flujo del material en las irregularidades que se presentan con gran frecuencia en el sistema de conductos radiculares $(26,27)$.

Sin embargo, en estudios de filtración con tintas se comprobó que la utilización del sistema Thermafil ${ }^{\circledR}$ con portadores (carriers) metálicos producía igual filtración que la técnica de condensación lateral en conductos curvos, a pesar de poseer un buen sellado en conductos rectos. Debido a estas discrepancias con el uso de portadores metálicos se desarrollaron portadores plásticos para la gutapercha blanda, los que han demostrado un mejor sellado apical (27).

Obturadores de Thermafil ${ }^{\circledR}$ con portadores de acero inoxidable y de titanio.

El sistema Thermafil ${ }^{\circledR}$ ha sacado al mercado tres diferentes tipos de portadores (carriers) para la gutapercha, los cuales son el acero inoxidable, titanio y plástico. Estos se encuentran cubiertos con gutapercha en fase alfa y forman parte de la obturación final. Se ha observado que la adaptación del sistema Thermafil ${ }^{\circledR}$ con portadores de plástico es mejor que la de los de acero y titanio, seguida la de los de titanio, siendo la peor la adaptación de los portadores de acero a las paredes del conducto debido a la contracción de la gutapercha dentro de las estrías del portador (28).

Obturadores de Thermafil ${ }^{\circledR}$ con portadores plásticos.

El sistema Thermafil ${ }^{\circledR}$ consiste en portadores plásticos (Thermafil Endodontic Obturators-plastic; Tulsa Dental Products, Tulsa, Oklahoma, USA) los cuales distribuyen la gutapercha más fácilmente y con exactitud considerable (26). El método original se ha modificado y evolucionado en lo que hoy se conoce como Thermafil Plus ${ }^{\circledR}$ (Dentsply, Tulsa Dental, Tulsa, OK), el cual está diseñado para obturar conductos instrumentados con limas rotatorias de níquel-titanio (Dentsply, Tulsa Dental). Recientemente ha introducido el sistema Thermafil 
GT® (Dentsply, Tulsa Dental), para la obturación de conductos preparados con el sistema de limas rotatorias GT (Dentsply, Tulsa Dental) (29).

Thermafil ${ }^{\circledR}$ consiste en un vástago portador central flexible, fabricado en tamaños y conicidades equivalentes a los de las limas endodónticas, recubierto de manera uniforme con una capa de gutapercha de la fase alfa refinada. La dificultad para llevarlo al conducto promovió una nueva modificación: actualmente estos vástagos son de plástico radiopaco. Los obturadores se comercializan en diferentes tamaños ISO y también hay obturadores Thermafil ${ }^{\circledR}$ adaptados a técnicas de instrumentación específicas, como GT Profile o el Protaper Universal (Dentsply Maillefer, Balagues, Suiza) con denominaciones F1, F2, F3, F4 y F5. Los vástagos de plástico, a pesar de tener el mismo aspecto y eficacia, no tienen la misma composición. Los de mayor diámetro, de 40 a 90 , se fabrican con polímero de polisulfona y por eso pueden disolverse en cloroformo, que en caso de retratamiento es de gran utilidad (1).

Basándose en un criterio radiológico, la obturación de conductos curvos con gutapercha alfa utilizando un portador plástico (Thermafil), resulta en un relleno más denso y mejor adaptado a lo largo de todo el conducto radicular, cuando se compara con la técnica de condensación lateral utilizando gutapercha estandarizada (beta) (28). Además se ha demostrado que tanto la condensación lateral como la técnica Thermafil ${ }^{\circledR}$ con portador plástico producen un sellado aceptable en el tercio apical del conducto; aunque existe una predisposición significativa de extrusión del mate- rial a través del ápice en la obturación con Thermafil ${ }^{\circledR}$ utilizando portadores plásticos $(26,27)$.

Se ha observado que el sistema Thermafil ${ }^{\circledR}$ provee un mejor sellado (estudios realizados con penetración de tinta) que la obturación con condensación lateral de conductos con una curvatura mayor a $25^{\circ}$. Lo que sugiere que el sistema Thermafil ${ }^{\circledR}$ provee mejor adaptación a las paredes de conductos curvos y que la técnica de condensación lateral se ve comprometida en conductos de mayor dificultad (30). Estos hallazgos confirman que la forma del conducto radicular y su curvatura afecta la calidad de la obturación (31), aunque otros autores han demostrado que no existe diferencia significativa en la obturación de un conducto curvo con la técnica de condensación vertical con gutapercha termo reblandecida y cuando se obtura con el sistema Thermafil ${ }^{\circledR}$ (32).

Una gran desventaja del sistema Thermafil ${ }^{\circledR}$ es la extrusión que produce a través del foramen apical, demostrándose una mayor extrusión con este sistema en comparación con condensación lateral y otros sistemas de gutapercha caliente (27). Esta extrusión depende, en gran medida de la velocidad de inserción del portador, una rápida inserción puede producir un sobre extensión de la gutapercha en tanto que una inserción lenta puede resultar en una subobturación (33,34). Al examinar la densidad de la obturación con estas dos técnicas, se ha observado que la densidad lograda es similar, con una tendencia para los obturadores de Thermafil ${ }^{\circledR}$ en producir una mayor calidad de la obturación en el tercio coronal; mientras que en el tercio apical es similar, aunque la calidad del sellado, es comparable en las dos técnicas (31).
Otro inconveniente del sistema Thermafil ${ }^{\circledR}$ consiste en el desalojo de la gutapercha del carrier plástico, lo que se puede explicar por la influencia de la curvatura del conducto radicular en el movimiento de la gutapercha blanda durante la colocación de esta. Sin embargo se ha demostrado que este sistema permite una buena adaptación del material de obturación a las irregularidades del conducto incluyendo los conductos accesorios $(27,34)$. La gutapercha, al utilizar el sistema Thermafil ${ }^{\circledR}$, es capaz de fluir dentro de los espacios laterales ó menores espacios, produciendo una mejor adaptación de la misma a la superficie radicular que cuando se emplea una técnica de condensación lateral (35).

$\mathrm{Al}$ estudiar los diferentes sistemas de obturación en cuanto a su sellado apical, se ha observado que al remover la gutapercha para hacer el espacio de un retenedor intrarradicular el sellado apical se ve comprometido. El sistema Thermafil ${ }^{\circledR}$ presenta una desventaja con respecto a su posterior desobturación para núcleo o retenedor intrarradicular, ya que se ha observado mayor microfiltración posterior a la elaboración de este espacio cuando se ha obturado previamente con Thermafil ${ }^{\circledR}$. Estos mismos resultados han sido obtenidos por Ravanshad y Torabinejad cuando demostraron que cuando se obtura con Thermafil ${ }^{\circledR}$ se produce más microfiltración después de hacer el espacio para un pin intraradicular que cuando se obtura con la técnica de condensación lateral o con la técnica de condensación vertical (36).

\section{Sistema Soft-Core ${ }^{\circledR}$.}

Soft-Core ${ }^{\circledR}$ es la técnica de obturación de conductos con guta- 
percha plastificada de la casa comercial Septodont. El principio de esta técnica también compromete un obturador plástico radiopaco que está igualmente recubierto de gutapercha en fase alfa. El obturador plástico tiene las medidas estandarizadas por la ISO. Se pueden encontrar desde el número 20 al 100. El obturador adecuado a utilizar tiene que quedar perfectamente dentro del conducto radicular y la gutapercha debe de ser calentada en el horno Soft-Core ${ }^{\circledR}$. El obturador plástico es suficientemente flexible para utilizarlo en conductos curvos y funciona como un espaciador para la gutapercha, éste ocupa aproximadamente dos tercios del espacio dentro del conducto.

La conicidad de los obturadores plásticos es suficiente para ejercer presión lateral durante la inserción, presión necesaria para sellar los conductos laterales y otras aberraciones que se puedan encontrar en el sistema de conductos radiculares. El mango y la porción metálica del obturador es removida luego de la inserción dentro del conducto, eso dejara la parte plástica del obturador dentro del conducto.

El kit Soft-Core ${ }^{\circledR}$ trae un verificador para corroborar el tamaño del conducto preparado y así poder escoger el tamaño adecuado del obturador. Ambos, tanto el verificador como el obturador, traen un tope plástico para poder medir la distancia de trabajo e inserción dentro del conducto. La utilización del verificador es sumamente importante, en el único caso en el cual no es necesario es cuando la instrumentación se lleva a cabo con limas rotatorias de conicidad mayor del $4 \%$ o del $6 \%$, ya que los obturadores del sistema Soft-Core ${ }^{\circledR}$ vienen en esta conicidad, y si se utilizan limas de mayor conicidad, el obturador va a bajar con facilidad dentro del conducto (37).

\section{Sistema Herofill ${ }^{\circledR}$.}

Este sistema consiste en portadores plásticos cubiertos con gutapercha termoplastificada en fase alfa. El portador central posee un tamaño estandarizado según ISO y el sistema provee un verificador de tamaño que asegura la adecuada preparación del conducto y la selección del obturador correcto de Herofill Soft Core ${ }^{\circledR}$. Este sistema fue diseñado para complementar el sistema de preparación de limas rotatorias de níquel-titanio Hero 642. Los obturadores tienen una conicidad de $2 \%$ y están disponibles en tamaños de 20-100, y poseen una longitud de $25 \mathrm{~mm}$. El sistema consta también de un horno pequeño que permite el calentamiento de la gutapercha en los obturadores.

Boussetta et al. (38) evaluaron la microfiltración apical y la capacidad de sellado del sistema Herofill Soft Core ${ }^{\circledR}$ y lo compararon con una técnica de condensación lateral termomecánica de gutapercha en frío mostrando como resultados una alta filtración en la técnica de condensación lateral (22,28\%) en oposición al Herofill Soft Core ${ }^{\circledR}$ $(7,32 \%)$ y la compactación termomecánica (8,76\%).

Es importante destacar que existen muchas variaciones en cuanto a los resultados cuando se han comparado estas técnicas, otros autores concluyeron que la condensación lateral y varios métodos de condensación híbridos tenían menor filtración que el Herofill Soft Core ${ }^{\circledR}$ y establecieron una analogía entre el Herofill Soft-Core ${ }^{\circledR}$ y el Thermafil ${ }^{\circledR}$ en el cual son el mismo producto distribuido por casas comerciales diferentes. Además, también en estudios sobre el Thermafil ${ }^{\circledR}$ encon- traron mayor o menor filtración cuando lo compararon con diferentes técnicas de condensación estableciendo que todas estas discrepancias en la literatura pueden estar relacionadas con alguna variación en la preparación del conducto radicular (38).

\section{Successfil ${ }^{\circledR}$.}

El Successfil ${ }^{\circledR}$ (Coltene Higienic, Akron, OH, EEUU), también utiliza un portador con el centro sólido cubierto con gutapercha de la fase alfa. Pero en este caso, la gutapercha permanece en una jeringa que al ser calentada, puede colocarse en el portador, antes de insertarlo en el conducto. Los transportadores Successfil ${ }^{\circledR}$, fabricados con titanio o plástico radiopaco, se llevan hasta la profundidad del conducto correspondiente de gutapercha dentro de la jeringa y se vierten comprimiendo el émbolo. La técnica de uso tiene ventajas e inconvenientes similares a los del Thermafil ${ }^{\circledR}(1,12)$.

\section{Simplifill ${ }^{\circledR}$.}

Un sistema de obturación con transportador rígido y ligeramente diferente es el Simplifill ${ }^{\circledR}$ (Lightspeed Technologies, San Antonio, TX, EEUU), ideado para el sistema de instrumentación Lighspeed ${ }^{\circledR}$, consistente en un vástago recubierto de gutapercha en sus últimos $5 \mathrm{~mm}$. Se selecciona una vástago que corresponda a la limamemoria (instrumento de mayor diámetro que alcanzo la longitud real de trabajo) y después de colocar el cemento en el conducto, se lleva el vástago a su interior con presión firme. Al llegar a la longitud real de trabajo se retira el vástago con un giro anti horario rápido, de hasta cuatro vueltas (1).

Según el fabricante puede completarse el resto de la obturación del 
conducto, solo con cemento a base de resina o con otra técnica de obturación (compactación vertical, gutapercha termo inyectada, termocompactación condensación lateral activa o la que se considere más oportuna). Es un sistema de obturación con eficacia de sellado similar a la de los demás (39).

\section{Conclusiones}

- El uso actual de las técnicas con gutapercha termo-plastificada permite al operador lograr un llenado tridimensional de todo el conducto radicular, siempre y cuando conozca las características y manejo de la gutapercha en su estado alfa y amorfo.

- El mejor sistema de obturación a emplear es aquel que ha sido elegido de acuerdo a la anatomía del conducto radicular a tratar, a la técnica que se utilice para su instrumentación, así como la habilidad y experiencia del operador para su uso.

- La hibridización entre las diversas técnicas de obturación nos permiten alcanzarlos objetivos principales de una obturación ideal; un correcto sellado apical y el llenado tridimensional de la totalidad del conducto radicular.

- Son necesarios mayores estudios en la técnica de conductores de centro sólido para evaluar su éxito a largo plazo, su grado de filtración y su dureza al compararlo con otras técnicas actuales.

\section{Referencias bibliográficas}

1. Leonardo MR, Leonardo RT. Endodoncia: Conceptos biológicos y recursos tecnológicos. Sao Paulo: Editorial Artes Médicas; 2009. p.91, 95

2. American Association of Endodontists: Appropriateness of care and quality assurance guidelines. In: Cohen S, Burns R. Vías de la Pulpa. 8 ed. Barcelona: Mosby; 2002.p.290-294.

3. Leonardo M, Leal J. Endodoncia. Tratamientos de los conductos. Editorial panamericana. Argentina 1994. p. 384 - 392.

4. Oguntebi BR, Shen C. Effect of different sealers on thermoplasticized Gutta-percha root canal obturations. J Endod. 1992; 18(8):363-6.

5. García Gonzales LA. Evaluación del sellado apical en obturaciones endodónticas utilizando sellador de mineral trióxido agregado [Tesis de Titulación]. Lima-Perú: Universisdad Nacional Mayor de San Marcos.

6. Gomes BP, Berber VB, Montagner F, Sena NT, Zaia AA, Ferraz CC, Souza-Filho FJ. Residual effects and surface alterations in disinfected guttapercha and Resilon cones. J Endod. 2007; 33(8):948-51.

7. Leonardo MR, Barnett F, Debelian GJ, de Pontes Lima RK, Bezerra da Silva LA. Root canal adhesive filling in dogs' teeth with or without coronal restoration: a histopathological evaluation. J Endod. 2007; 33(11):1299-303.

8. Shipper G, Ørstavik D, Teixeira FB, Trope M. An evaluation of microbial leakage in roots filled with a thermoplastic synthetic polymer-based root canal filling material (Resilon). J Endod. 2004; 30(5):342-7.

9. Shipper G, Teixeira FB, Arnold RR, Trope M. Periapical inflammation after coronal microbial inoculation of dog roots filled with gutta-percha or resilon. J Endod. 2005; 31(2):916.

10. Estrela C. Ciencia endodóntica. Sao Paulo: Editorial Artes Médi- cas; 2005. p.562.

11. Wu MK, van der Sluis LW, Wesselink PR. A preliminary study of the percentage of guttapercha-filled area in the apical canal filled with vertically compacted warm gutta-percha. Int Endod J. 2002; 35(6):527-35.

12.De-Deus G, Gurgel-Filho ED, Magalhães KM, Coutinho-Filho T. A laboratory analysis of guttapercha-filled area obtained using Thermafil, System B and lateral condensation. Int Endod J. 2006; 39(5):378-83.

13. Guess GM, Edwards KR, Yang ML, Iqbal MK, Kim S. Analysis of continuous-wave obturation using a single-cone and hybrid technique. J Endod. 2003; 29(8):509-12.

14. Lea CS, Apicella MJ, Mines $P$, Yancich PP, Parker MH. Comparison of the obturation density of cold lateral compaction versus warm vertical compaction using the continuous wave of condensation technique. J Endod. 2005; 31(1):37-9.

15. Smith RS, Weller RN, Loushine RJ, Kimbrough WF. Effect of varying the depth of heat application on the adaptability of gutta-percha during warm vertical compaction. J Endod. 2000; 26(11):668-72.

16.Villegas JC, Yoshioka T, Kobayashi C, Suda H. Threestep versus single-step use of system B: evaluation of guttapercha root canal fillings and their adaptation to the canal walls. J Endod. 2004; 30(10):71921.

17. Coltene. roeko GuttaFlow ${ }^{\circledR 2 / ~}$ GuttaFlow ${ }^{\circledR}$ FAST [Internet]. Disponible en: http:// www.coltene.com/en/products/ $21 / \mathrm{d}$ e t a i l s/53/ Gutta F l ow \% C 2 \% A E - 
GuttaFlow\%C2\%AE_FAST.html

18. Lozano B, Reyes J, Garrido P, Mena J, Vera C, Rodríguez N. Estudio comparativo del grado de filtración del cemento Guttaflow ${ }^{\circledR}$ según técnica estándar versus condensación lateral. Cient Dent [Internet]. 2009; 6(3):223-30. Disponible en: http://www.coem.org.es/sites/ default/files/revista/cientifica/ vol6-n3/67-74.pdf.

19. Grassi MD, Plazek DJ, Michanowicz AE, Chay IC. Changes in the physical properties of the Ultrafil lowtemperature (70 degrees $\mathrm{C}$ ) thermoplasticized gutta-percha system. J Endod, v.15, n.11, p.517-521, 1989.

20. Harris GZ, Dickey DJ, Lemon RR, Luebke RG. Apical seal: McSpadden vs lateral condensation. J Endod, v.8, n.6, p.273-276, 1982.

21. Kersten HW, Fransman R, Thoden van Velzen SK. Thermomechanical compaction of gutta-percha II. A comparison with lateral condensation in curved root canals. Int Endod J, v.19, n.3, p.134-140, 1986.

22.Lipski M. Root surface temperature rises in vitro during root canal obturation using hybrid and microseal techniques. J Endod. 2005; 31(4):297-300.

23. Lipski M. In vitro infrared thermographic assessment of root surface temperatures generated by high-temperature thermoplasticized injectable gutta-percha obturation technique. J Endod. 2006; 32(5):438-41.

24. Lipski M, Wozniak K. In vitro infrared thermographic assessment of root surface temperature rises during thermafil retreatment using system B. J Endod. 2003; 29(6):413-5.

25. Tagger M, Tamse A, Katz A, Korzen BH. Evaluation of the apical seal produced by a hybrid root canal filling method, combining lateral condensation and thermatic compaction. J Endod, v.10, n.7, p.299-303, 1984.

26.Gutmann JL, Saunders WP, Saunders EM, Nguyen L. An assessment of the plastic Thermafil obturation technique. Part 1. Radiographic evaluation of adaptation and placement. Int Endod J. 1993; 26(3):173-8.

27.Gutmann JL, Saunders WP, Saunders EM, Nguyen L. An assessment of the plastic Thermafil obturation technique. Part 2. Material adaptation and sealability. Int Endod J. 1993; 26(3):179-83.

28. Weller RN, Kimbrough WF, Anderson RW. A comparison of thermoplastic obturation techniques: adaptation to the canal walls. J Endod. 1997; 23(11):703-6.

29. Robinson MJ, McDonald NJ, Mullally PJ. Apical extrusion of thermoplasticized obturating material in canals instrumented with Profile 0.06 or Profile GT. J Endod. 2004; 30(6):418-21.

30. Leung SF, Gulabivala K. An invitro evaluation of the influence of canal curvature on the sealing ability of Thermafil. Int Endod J. 1994; 27(4):190-6.

31. Dummer PM, Lyle L, Rawle J, Kennedy JK. A laboratory study of root fillings in teeth obturated by lateral condensation of guttapercha or Thermafil obturators. Int Endod J. 1994; 27(1):32-8.

32. Fan B, Wu MK, Wesselink PR.
Leakage along warm gutta-percha fillings in the apical canals of curved roots. Endod Dent Traumatol. 2000; 16(1):29-33.

33.Levitan ME, Himel VT, Luckey JB. The effect of insertion rates on fill length and adaptation of a thermoplasticized gutta-percha technique. J Endod. 2003; 29(8):505-8.

34. Chu CH, Lo EC, Cheung GS. Outcome of root canal treatment using Thermafil and cold lateral condensation filling techniques. Int Endod J. 2005; 38(3):179-85.

35.Clinton K, Van Himel T. Comparison of a warm guttapercha obturation technique and lateral condensation. J Endod. 2001; 27(11):692-5.

36.Fan B, Wu M-K, Wesselink PR. Leakage along warm gutta-percha fillings in the apical canals of curved roots. Endodont Dent traumatol, 2000: 1629 - 33.

37. Sistemas de Obturación con Gutapercha Termoplastificada [Internet]. Disponible en: http:// www.javeriana.edu.co/ academiapgendodoncia/ art_revision/revision_2006/ i_a_revision31.html

38. Boussetta F, Bal S, Romeas A, Boivin G, Magloire H, Farge P. In vitro evaluation of apical microleakage following canal filling with a coated carrier system compared with lateral and thermomechanical Gutta-Percha condensation techniques. Int Endod J. 2003; 36(5):367-71.

39. Santos MD, Walker WA 3rd, Carnes DL Jr. Evaluation of apical seal in straight canals after obturation using the Lightspeed sectional method. J Endod. 1999; 25(9):609-12. 